\title{
Development of ECE/ECEI diagnostics and MHD-related studies on HL-2A tokamak
}

\author{
Z.C. Yang ${ }^{1}$, M. Jiang ${ }^{1, *}$, Z.B. Shi ${ }^{1}$, X.T. Ding ${ }^{1}$, N.C. Luhmann, Jr. ${ }^{2}$, W.L. Zhong ${ }^{1}$, W. Chen ${ }^{1}$, P.W. Shi ${ }^{1}$, Y. Xu ${ }^{3}$, J. Wen ${ }^{1}$, \\ A.S. Liang ${ }^{1}$, Y. Liu ${ }^{1}$, Q.W. Yang ${ }^{1}$ and HL-2A team \\ ${ }^{1}$ Southwestern Institute of Physics, P. O. Box 432, Chengdu 610041, China. \\ ${ }^{2}$ Department of Electrical and Computer Engineering, University of California, Davis, California 95616, USA \\ ${ }^{3}$ Institute of Fusion Science, School of Physical Science and Technology, Southwest Jiaotong University Chengdu 610031, China
}

\begin{abstract}
A novel 60-channel electron cyclotron emission (ECE) radiometer has been designed and tested for the measurement of electron temperature profiles on the HL-2A tokamak. This system is based on the intermediate frequency division technique, and has the features of wide working frequency range $(60-90 \mathrm{GHz})$ and high temporal-spatial resolution $(3 \mu \mathrm{s}, 1 \mathrm{~cm})$, which covers almost the entire plasma region. Also, an electron cyclotron emission imaging (ECEI) system has been developed for studying two dimensional electron temperature fluctuations. It is comprised of several front-end quasi-optical lenses, a 24 channel heterodyne imaging array with a tunable RF frequency range spanning $60-135 \mathrm{GHz}$, and a set of back-end ECEI electronics that together generate two $24 \times 8$ array images of the 2 nd harmonic X-mode electron cyclotron emission from the HL-2A plasma. The measurement region can be flexibly shifted due to two independent local oscillator sources, and the field of view can be adjusted easily by changing the position of the zoom lenses as well. The temporal resolution is about $2.5 \mu \mathrm{s}$ and the achievable spatial resolution is $1 \mathrm{~cm}$. The ECE/ECEI diagnostics have been demonstrated to be powerful tools to study MHD-related physics including the multi-scale interaction between macro-scale MHD and micro-scale turbulence on the HL-2A tokamak.
\end{abstract}

\section{Introduction}

Electron temperature $\left(T_{e}\right)$ is one of the basic parameters in fusion plasma, and its measurements are essential for successful completion of the fusion device mission. At present, it can be diagnosed in various ways, including laser Thomson scattering, soft x-ray using silicon drift detector, Langmuir probes, and electron cyclotron emission (ECE). However, for Thomson scattering the time resolution is about several to tens of millisecond, and for the Langmuir probe, the measurement can only reach the edge region. The interpretation for silicon drift detector measurements are tricky since they depend on the electron temperature, density and impurity. In contrast, ECE diagnostic is an effective method to determine the $T_{e}$ profile and $T_{e}$ fluctuation $\tilde{T}_{e}$ with good temporal and spatial resolution, and therefore would become a routine tool to detect $T_{e}$ evolution in ITER [1]. ECE electron temperature data will be important for evaluating the radial deposition profile of EC heating and the time evolution of the alpha particle heating. Furthermore, real-time ECE measurements will be used to identify magnetohydrodynamic (MHD) modes, such as neoclassical tearing modes that can often be deleterious to plasma performance.

Under the condition of optically thick emission, the $2^{\text {nd }}$ harmonic of ECE can be considered as Blackbody emission, which is described by Planck's law in the Rayleigh-

*e-mail: jiangm@swip.ac.cn
Jeans limit. Consequently, the intensity of ECE for the optically thick case is expressed by Eq.1 [2, 3].

$$
I_{E C E}=\frac{\omega^{2} T_{e}}{8 \pi^{2} c^{2}}=C T_{e}
$$

By calibrating the coefficient $\mathrm{C}$, the $T_{e}$ profile can be obtained. The previous ECE radiometers on HL-2A tokamak had a finite working frequency range which limits the available measurement coverage in the plasma especially when the toroidal magnetic field changes. In addition, the spatial resolution was about $2-3 \mathrm{~cm}$, which makes it difficult to identify the accurate locations of some events, such as ECH deposition, sawtooth inversion radius, and tearing modes. Also, the linear operating range needs to be extended to satisfy the higher heating power condition during the discharges. Hence, a novel 60 channel ECE radiometer has been developed to measure the $T_{e}$ profile of the entire plasma region with a spatial resolution of $0.5-1 \mathrm{~cm}$. The detailed introduction of this system will be given in Section 2. The traditional 1D ECE diagnostic measures the electron temperature locally at different radii along midplane of the device [4]. To satisfy the need for multidimensional MHD physics measurements, the 2D electron cyclotron emission imaging (ECEI) technique has been developed by extending the traditional ECE approach by incorporating large aperture quasi-optical lenses and vertically aligned antenna arrays. The detailed description of ECEI on HL-2A and the optical improvements will be pre- 
sented in Section 3. In Section 4, two examples regarding MHD-related studies by using ECE/ECEI are presented. Last, a summary is presented in Section 5.

\section{60-channel ECE with intermediate frequency division technique}

FIG.1 shows the schematic of the newly developed 60channel ECE diagnostic. Compared to the previous ECE radiometers, this system is based on the direct intermediate frequency (IF) filtering and detection technique by using several multiplexers instead of the second frequency down-converting (mixing) and power division technique [5]. Note that the power divider will introduce large insertion loss and is quite expensive especially at high frequency. This direct frequency filtering technique has three advantages: lower cost, wider working frequency range, and higher IF output power. The local oscillator (LO) source can be tunable between 50 and $90 \mathrm{GHz}$ at a power level of $10 \mathrm{dBm}$, and the ECE signal from plasma is down converted to an IF signal with a frequency of 1-33 GHz. In the IF modules, the signal is divided by a 2way resistive-type power divider, and then amplified by the wide-band low noise amplifier (LNA) separately. After that, the signals are split into 60 channels ( 2 to 31.5 $\mathrm{GHz}$ with a step of $0.5 \mathrm{GHz}$ and bandwidth of $0.5 \mathrm{GHz}$ ) by eight-frequency multiplexers, and then detected, video amplified and low pass filtered with a bandwidth of 300 $\mathrm{kHz}$.

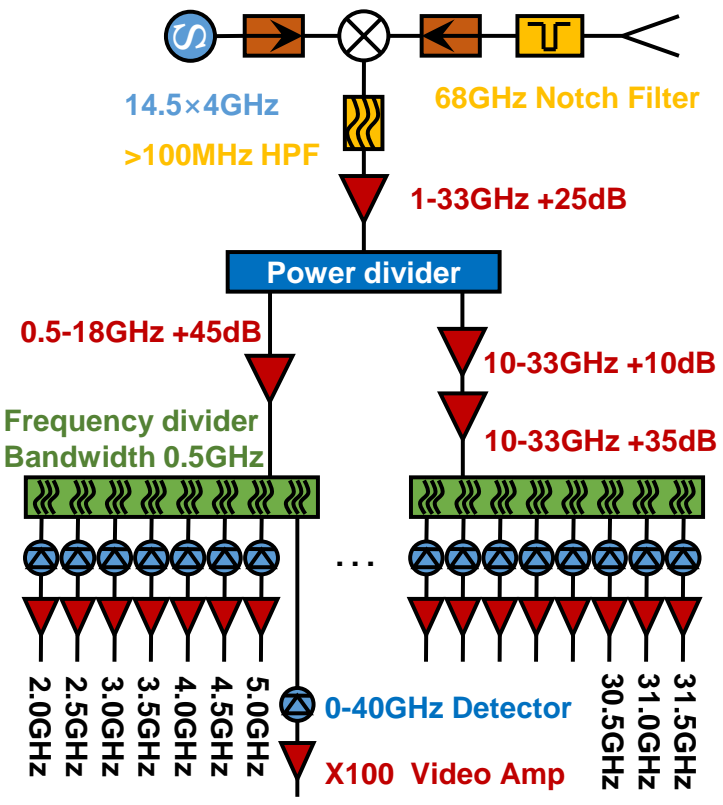

Figure 1. Schematic of the newly developed 60-channel ECE radiometer.

The system was tested in the laboratory before installation on the HL-2A tokamak. Figure 2(a) shows the frequency response of the LNA used in the IF module after the two-way power divider as illustrated in Fig.1. The LNA can amplify IF signals by $30-40 \mathrm{~dB}$ over the whole frequency band. The high IF output power improved the signal to noise ratio significantly. Shown in Fig.2(b) is the frequency response of the filter for each IF channel. The frequency interval between adjacent channels is $500 \mathrm{MHz}$ and the bandwidth of the filters is also $500 \mathrm{MHz}$. The test result shows that there is almost no overlap between neighboring channels, and a relatively flat response is obtained over the full working band. The utilization of the narrow band filter guaranteed a high spatial resolution of $T_{e}$ measurement, i.e., $\sim 1 \mathrm{~cm} / 0.5 \mathrm{~cm}$ in the $B_{t}=1.3 / 2.4 \mathrm{~T}$ operation. Fig. 2(c) shows the power response of a detector with $f_{I F}=23 \mathrm{GHz}$. The detector works over a very wide linear region with input IF power less than $1.1 \mu \mathrm{W}$ and output power within $-2 \mathrm{~V}-2.5 \mathrm{~V}$. In this case, the detector output voltage is proportional to the electron temperature. Base on the system performance introduced above, the ECE system can provide reliable profiles even with megawatt electron cyclotron resonance heating (ECRH). For the routine discharge on the HL-2A tokamak, the measurement range will cover about $66 \mathrm{~cm}$ on the midplane, with a spatial resolution of $1.1 \mathrm{~cm}$ and temporal resolution of $3 \mu \mathrm{s}$.

\section{ECEI on HL-2A and the optical optimization}

Although the 1D ECE measurement can provide $T_{e}$ profile with high temporal and spatial resolution, it cannot provide the global quantities of the instabilities in the plasma, due to the fact that the MHD physics is always multidimensional. To capture the $2 \mathrm{D}$ characteristic of the MHD mode, we have developed two $24 \times 8$ ECEI array under the collaboration with University of California, Davis. ECEI is an advanced extension of the traditional ECE radiometry with the same basic principle; however, it can image the plasma onto a vertically aligned antenna array by using a set of quasi-optical lenses with the heterodyne frequency capability yielding 2D images. The ECEI diagnostic has been proven to be a powerful tool for MHD physics through electron temperature fluctuation measurements, providing invaluable images on core/edge physics [6-11]. The ECEI instrument includes four subsystems, i.e., quasi-optical system, antenna array, microwave receivers and digitizers. The detailed introduction of each subsystem, its laboratory calibration, upgrade and preliminary experimental results are described in previous publications $[12,13]$. According to different physical requirements, the system can work in narrow zoom pattern (higher spatial resolution) and wide zoom pattern (larger spatial coverage) by adjusting the relative positions of the zoom lenses. Using two separate LO sources, the measurement regions of the two $24 \times 8$ ECEI arrays can be shifted flexibly in the radial direction. It has 16 channels in the horizontal direction and 24 channels in the vertical direction, covering a rectangular area of $40-55 \mathrm{~cm}$ (vertical) $\times$ $12-17 \mathrm{~cm}$ (radial). The temporal and spatial resolutions are $2.5 \mu \mathrm{s}$ and $1-3 \mathrm{~cm}$, respectively.

In the laboratory calibration and plasma experiments, we found that the quasi-optical system had some limitations: (i) the focal area can only reach the core and low 

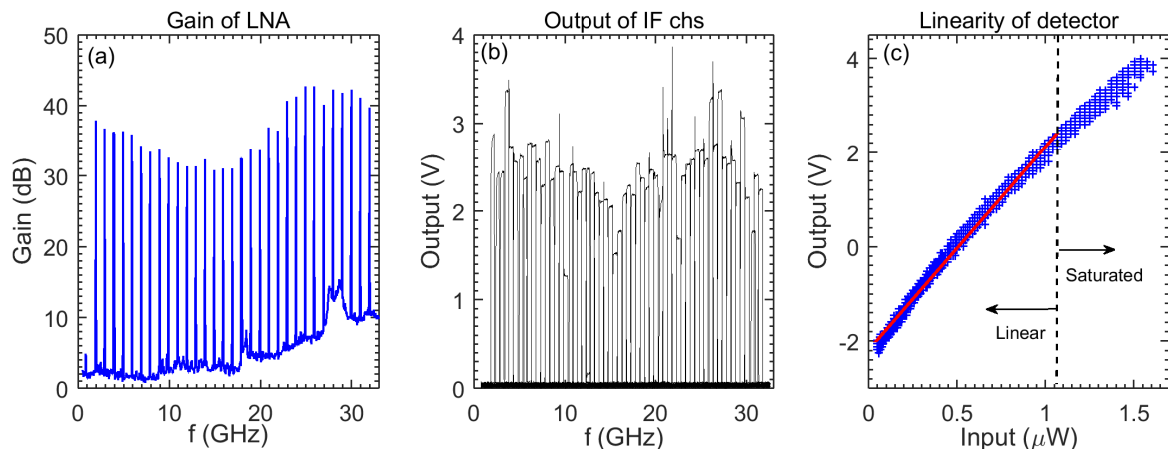

Figure 2. (a) the gain of the wide-band low noise amplifier in the IF module, (b) the frequency response of each IF channel, and (c) the power response of the detector.

field side (LFS) region of the plasma, but not the high field side (HFS) region; (ii) the focal plane is very curvy. The curved focal plane indicates that the edge vertical channels are out of focus, leading to a low signal to noise ratio of the signals. Consequently, last year, we have optimized the quasi-optical system to obtain better focal performance by changing the curvature radius of the focal lens, the surface type of the zoom lenses, and the relative distance between the lenses. Figure 3 shows the results of the Gaussian beam tracing of the radio frequency optics before and after optimization. The focal lens, zoom lenses, and the distance between the antenna and the focal lens (L) are marked in the figure. The zoom lenses are used to adjust the coverage of the vertical field of view. By moving the focal lens, the radial location of the focal plane can be shifted. It can be seen that before the modification of the optics, the focal plane is very curvy, while after the modification it becomes much flatter.

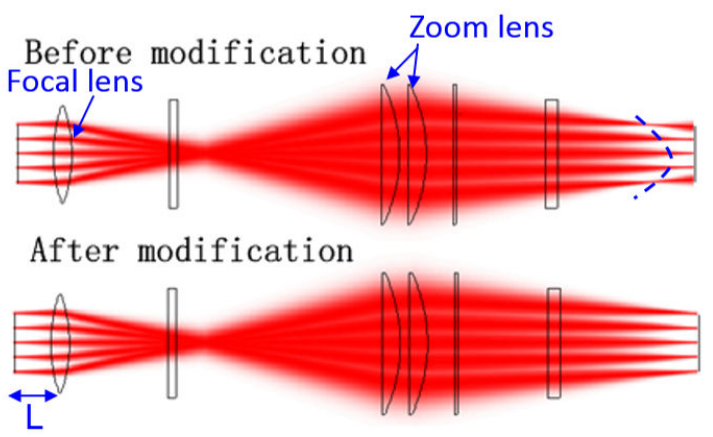

Figure 3. Gaussian beam tracing of the radio frequency optics before and after the optics modification.

Shown in Figure 4 are distributions of the focal planes with different $L$ values under narrow zoom and wide zoom patterns, calculated from the real ray tracing. In the wide zoom pattern, the vertical field of view is larger than that in the narrow zoom pattern. It was found that in both narrow and wide zoom patterns, the field curvature of the focal plane ( $\triangle R$ between the core channel and the edge channel) is within $6 \mathrm{~cm}$. In addition, the focal area can reach the high field side plasma region. The optimized optics were fabricated, tested and finally installed on the HL-2A tokamak for the 2018 spring experimental campaign. The quality of the ECEI signals were highly improved in this experiment.
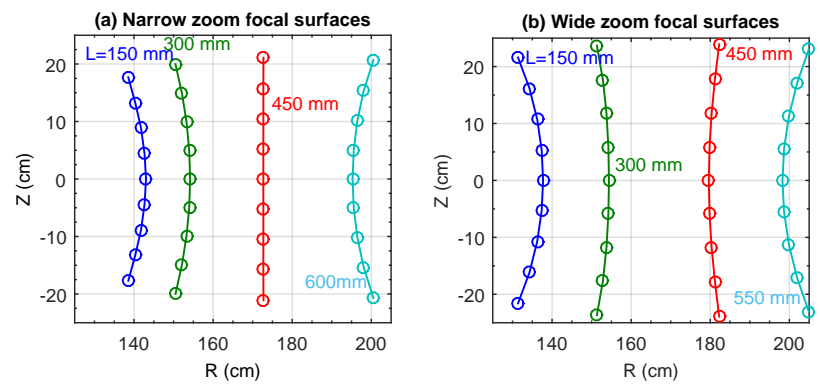

Figure 4. Focal plane with different positions of focal lens under narrow zoom and wide zoom patterns.

\section{MHD-related studies by ECE/ECEI}

\subsection{Calibration of the ECE system}

Normally, due to the different electronic response of each channel, the ECE measurement has to be calibrated to represent the $T_{e}$ profile. As we know, the detection locations of ECE depend on the working frequency and magnetic field. Hence, the ECE radiometer can be relatively calibrated by changing the working frequency or magnetic field slightly in two similar discharges with the assumption of a stiff $T_{e}$ profile. The absolute values of the $T_{e}$ can be obtained through cross comparison with the core Thomson scattering data. The calibration method by hopping working frequency has been introduced in [5]. Here we show one example of the ECE calibration by shifting the magnetic field, which is the routine way to obtain $T_{e}$ profiles in HL-2A. The calibrated $T_{e}$ profiles are shown in figure 5(a). As we mentioned in Section 2, this ECE radiometer can work in a wider, linear input region compared with previous systems; it has been verified that even under high power ECRH the system can still work well. Fig. 5(b) indicates that with on-axis ECRH, the plasma center shifted outward by $5 \mathrm{~cm}$ and the core $T_{e}$ was increased by $150 \%$ compared to the ohmic heating case. 

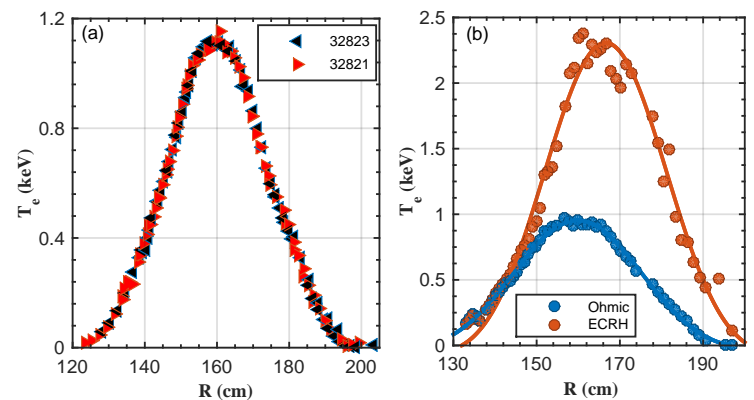

Figure 5. (a) Calibrated $T_{e}$ profiles obtained by shifting the magnetic field in two similar discharges, and (b) $T_{e}$ profiles under ohmic and ECRH heating conditions.

\subsection{Analysis of radial mode structure}

The newly developed ECE system basically covers $90 \%$ of the plasma along the midplane with a spatial resolution of $\sim 1 \mathrm{~cm}$. Consequently, it represents a good tool to detect the radial structure of MHD modes, such as the accurate location and width of the tearing mode (TM). In the experiment, the mode rotates past the ECE detector position, so ECE signal variations can be used to distinguish between kink and tearing modes. Plotted in figure 6 is a representative comparison of the features between the $m / n=2 / 1 \mathrm{TM}$ and the $m / n=1 / 1$ ideal kink mode. Fig. 6(a) illustrates the time history of the $T_{e}$ located inside, but near the $q=2$ surface, and Fig. 6(e) shows $T_{e}$ near the $q=1$ surface. The ideal kink mode produces sinusoidal temperature variation near the resonant surface (see Fig. 6(e)), while for the tearing mode, due to the flat profile near the O-point of the island, the $T_{e}$ variation near the resonant surface will be deformed when the island structure passes by (see Fig. 6(a)), which is clearly illustrated by the cartoon in Reference [14]. For the position inside, but near the $q=2$ surface, the more flattened trough of the $T_{e}$ variation indicates that the $\mathrm{O}$-point of the island is across the probed location (blue vertical line), while the more peaked crest means the $\mathrm{X}$-point is through there (red vertical line).

Figures 6(b), 6(c), 6(f) and 6(g) show the amplitude and phase of relative electron temperature variations $\left(\delta T_{e} / \bar{T}_{e}\right)$ using fast Fourier transform analysis for TM and ideal kink mode, respectively. The shaded area illustrates the locations of the modes. The TM mode is located at 136 $\mathrm{cm}<R<144 \mathrm{~cm}$ in the HFS and $180 \mathrm{~cm}<R<188 \mathrm{~cm}$ in the LFS, which corresponds to the two peak areas in Fig. 6(b) and the flat $T_{e}$ profile with the O-point of island passing by (blue circles in Fig. 6(d)). For the 1/1 ideal kink mode, the mode structure is encompassed by the $q=1$ surface, which is $R=150 \mathrm{~cm}$ in the HFS and $R=180 \mathrm{~cm}$ in the LFS. The $T_{e}$ profile is not flattened for the kink mode (see Fig. 6(h)), demonstrating that there is no magnetic reconnection. In addition, for TM $T_{e}$ perturbation takes on the same phase $(2 \pi)$ between the HFS and LFS (see Fig. 6(c)), consistent with the even behavior of $m=2$, whereas for the internal kink mode an opposite phase associated with odd structure was observed (see Fig. 6(g)), in agreement with $m=1$. It was also found that the core $T_{e}$ profile becomes
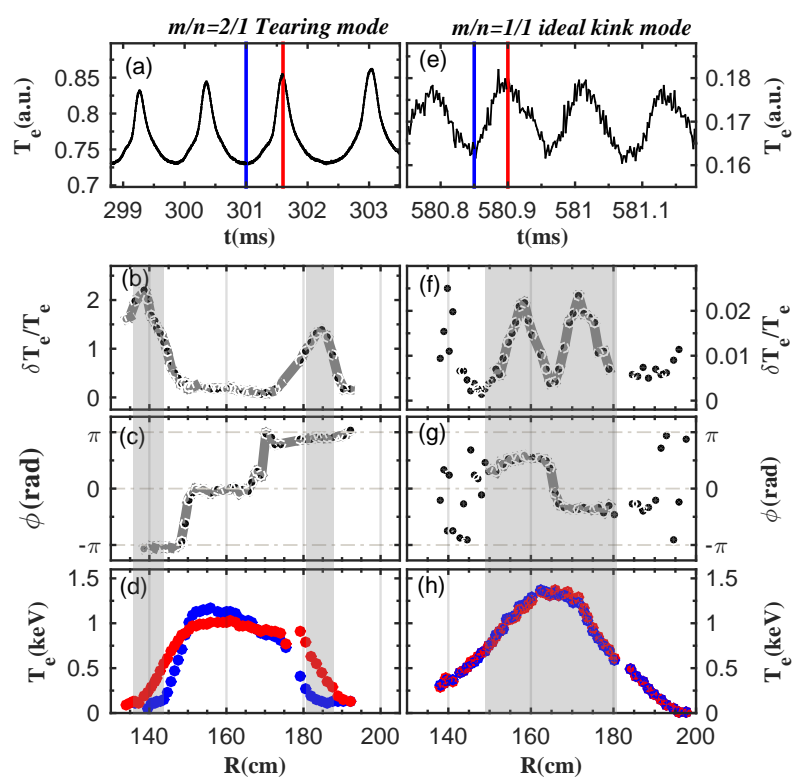

Figure 6. (a) time evolution of $T_{e}$ inside but near the $q=2$ surface, (b-c) the amplitude and phase of the relative $T_{e}$ variation $\delta T_{e} / \bar{T}_{e}$ with an $m / n=2 / 1$ tearing mode, (d) $T_{e}$ profiles at two moments (O-/X-point passing-by) marked by the blue and red vertical lines in (a), (e) time evolution of $T_{e}$ near the $q=1$ surface, (f-g) the amplitude and phase of the relative $T_{e}$ variation $\delta T_{e} / \bar{T}_{e}$ with an $m / n=1 / 1$ ideal kink mode, and (h) $T_{e}$ profiles at two moments marked by the blue and red vertical lines in (e). The locations of the mode were shaded in the figure.

much flatter when there exists a TM compared to without TM cases, probably due to the impurity accumulation in the core plasma region.

\subsection{Multi-scale interaction between TM and turbulence}

In HL-2A, the ECEI diagnostic has been extensively used to detect the 2D structure of large scale MHD modes $[10,11,15,16]$, such as tearing mode, fishbone, and sawtooth crash. Figure 7 shows the mode structure of the $m / n=2 / 1$ tearing mode. The phase of $T_{e}$ variations is opposite on two sides of the $q=2$ surface. The X-point and $\mathrm{O}$-point of the island are indicated with the black $\mathrm{X}$ and $\mathrm{O}$ marks in the figures 7(a) and 7(b), respectively. Recently, multi-scale physics such as the interaction between magnetic islands and turbulence was found to play an important role in regulating the turbulent transport [15, 17-23], and therefore has become an important topic in fusion research. ECEI was demonstrated to be a powerful tool for analyzing such multi-scale physics. On the one hand, from the ECE images, the rotation frequency and direction, X/O-point, the location and width of the island can be identified accurately. On the other hand, it can also provide information of high frequency $T_{e}$ fluctuations, $\tilde{T}_{e}$.

Figure 8 is a typical example of the interaction between the magnetic island and $T_{e}$ turbulence. In Fig. 8(a), the power spectrum of raw signals (passing through the island) measured by ECEI is plotted. The coherent peak at 
$f \approx 4.3 \mathrm{kHz}$ indicates the modulation on the local electron temperature by the island rotation. To gain further insight into the effect on broadband turbulence, we analyzed the envelope of temperature fluctuations $\left(\xi_{\tilde{T}_{e}}(t)\right)$ in the frequency range of $20-100 \mathrm{kHz}$ (see shaded areas), i.e., $\xi_{\tilde{T}_{e}}(t)=\left(\frac{1}{2 \pi} \int_{20 k H z}^{100 k H z} \tilde{T}_{e}(f) e^{i 2 \pi f t} d f\right)^{2}$, where $\tilde{T}_{e}(f)$ is the Fourier transform of $\tilde{T}_{e}(t)$. The frequency spectra of $\xi_{\tilde{T}_{e}}(t)$ is shown in the inset of Figs. 8(a). The envelope spectrum exhibits clear peaks at the rotating TM frequency, signifying the modulation of broadband turbulence by rotating islands. Fig. 8(b) further display the time traces of $\tilde{T}_{e}$ (integrated from $20-100 \mathrm{kHz}$ ), where the envelope of $\tilde{T}_{e}$ was calculated via the Hilbert transform and depicted by the red curves. The amplitude in $\tilde{T}_{e}$ is modulated during the rotation of the island, i.e. minimum at the O-point and maximum at X-point, which is consistent with gradientdriven turbulence. Furthermore, using two vertically adjacent ECEI channels, the poloidal flow velocity could be deduced from the vertical pattern velocity. Thus, the poloidal flow shear in the island region can be investigated. It was reported on KSTAR that the combined effects of the $T_{e}$ gradient and the poloidal flow shear determine the twodimensional distribution of the $T_{e}$ turbulence [22].
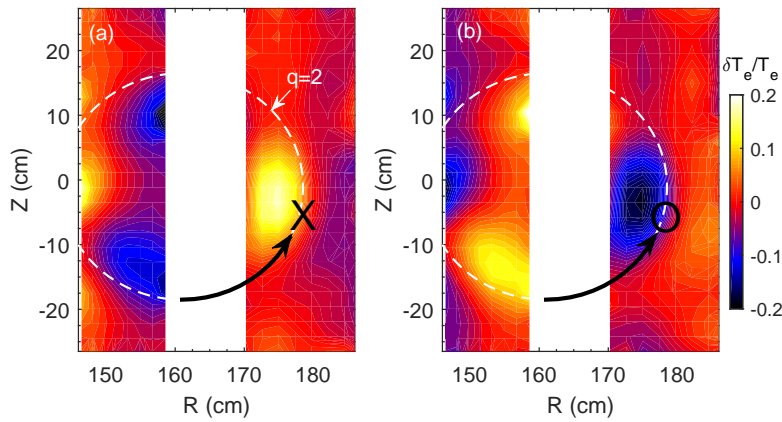

Figure 7. 2D mode structure of the $m / n=2 / 1$ tearing mode measured by ECEI. The white dashed curves denote the $q=2$ surface, and the X-point and O-point of the island are indicated with the black $\mathrm{X}$ and $\mathrm{O}$ marks, respectively.

\section{Summary}

A novel 60-channel ECE radiometer has been developed for measuring $T_{e}$ profile and $T_{e}$ variations on the HL-2A tokamak. It is based on the direct intermediate frequency division technique by utilizing several multiplexers which ensures high IF output power, and hence high signal to noise ratio of the signals. The measurements of this system can cover almost $90 \%$ of the plasma along the midplane, and the temporal and spatial resolutions are $3 \mu \mathrm{s}$ and $1 \mathrm{~cm}$ for the routine $B_{t}=1.3 \mathrm{~T}$ operation. Two $24 \times 8$ ECEI arrays have been developed by incorporating quasioptical lenses and vertical aligned antenna array for two dimensional $\tilde{T}_{e}$ measurements. The rectangular coverage of the two arrays can be flexibly shifted due to two independent LO sources, and the field of view can be adjusted easily by changing the position of the zoom lenses as well.
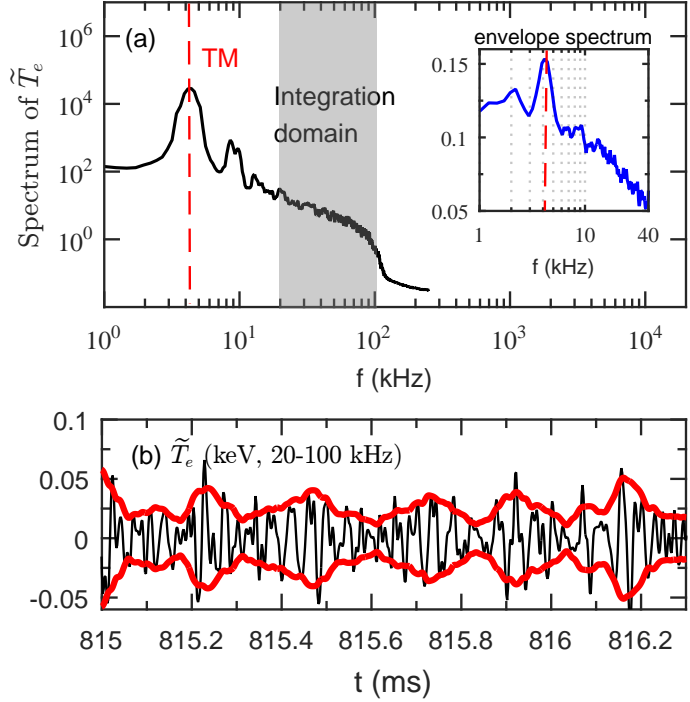

Figure 8. (a) Power spectrum of $\tilde{T}_{e}$ measured at $R=182.14 \mathrm{~cm}$ and $Z=3.46 \mathrm{~cm}$. The inset in (a) shows the amplitude spectrum of the corresponding envelope signal, $\xi_{\tilde{T}_{e}}(t)$ in the frequency range $\Delta f=20-100 \mathrm{kHz}$ (shaded in (a)). (b) Time histories of $\tilde{T}_{e}$ (integrated from $20-100 \mathrm{kHz}$ ) and the envelopes of $\tilde{T}_{e}$ depicted by the black and red curves, respectively.

The time and maximum spatial resolutions are $2.5 \mu$ s and $1 \mathrm{~cm}$, respectively. The optics of ECEI has been modified to cover broader plasma region and improve the imaging quality. The broad coverage and high spatial resolution of the ECE make it a powerful tool to study MHD-related physics, such as distinguishing the tearing mode from the kink mode, the location and width of magnetic island. In addition, multi-scale interaction between TM and high frequency turbulent $\tilde{T}_{e}$ has been investigated by ECEI diagnostic.

This work is partially supported by the National Natural Science Foundation of China under grant No. 11705051, 11475057, 11775069 and 11705049, partially supported by National Key R\&D Program of China (Grant Nos. 2017YFE0301201 and 2017YFE0301203), and partially supported by Sichuan Science and Technology Program (Grant No. 2018RZ0123).

\section{References}

[1] G. Taylor, et al., EPJ Web of Conferences 87, 03002 (2005)

[2] M. Bornatici, et al., Nucl. Fusion 23, 1153 (1983)

[3] I. H. Bornatici, Principles of Plasma Diagnostics (Cambridge University Press, Cambridge,1987), Chapter 5 .

[4] H. J. Hartfuss, T. Geist and M.Hirsch, Plasma Phys. Controlled Fusion 39, 1693 (1997)

[5] Z.B. Shi, et al., Rev. Sci. Instrum. 85, 023510 (2014)

[6] H.K. Park, et al., Phys. Rev. Lett. 96, 195003 (2006)

[7] I.G.J. Classen, et al., Phys. Rev. Lett. 98, 035001 (2007) 
[8] I.G.J. Classen, et al., Plasma Phys. Controlled Fusion 53, 124018 (2011)

[9] J. Lee, et al., Phys. Rev. Lett. 117, 075001 (2016)

[10] M. Jiang, et al., Phys. Plasmas 24, 022110 (2017)

[11] M. Jiang, et al., Nucl. Fusion 58, 026002 (2018)

[12] M. Jiang, et al., Rev. Sci. Instrum. 84, 113501 (2013)

[13] M. Jiang, et al., Rev. Sci. Instrum. 85, 076107 (2015)

[14] V. Igochine et al., Nucl. Fusion 57036015 (2017)

[15] W. Chen et al., Nucl. Fusion 57114003 (2017)
[16] L.M. Yu et al., Nucl. Fusion 57036023 (2017)

[17] Bardóczi L. et al Phys. Rev. Lett. 116215001 (2016).

[18] Bardóczi L. et al Phys. Plasmas 24056106 (2017).

[19] Ji X. Q. et al Sci. Rep. 632697 (2016).

[20] Ida K. et al Sci. Rep. 516165 (2015).

[21] Zhao K. J. et al Nucl. Fusion 57126006 (2017).

[22] Choi M. J. et al Nucl. Fusion 57126058 (2017).

[23] Sun P. J. et al Nucl. Fusion 58016003 (2018). 